\title{
Albumin: the next-generation delivery technology
}

\author{
"It is clear there is a need for next-generation half-life extension and \\ targeted drug-delivery technologies that are simple in design, \\ biodegradable and nonimmunogenic.
}

\begin{abstract}
Keywords: albumin $\bullet$ albumin-binding $\bullet$ albumin fusions $\bullet$ half-life extension $\bullet$ human serum albumin $\bullet$ intracellular delivery $\bullet$ neonatal Fc receptor (FcRn) • recombinant albumin - targeted drug-delivery
\end{abstract}

\section{Motivation for next-generation delivery technology}

The therapeutic efficacy of a drug is determined by the ability for site-specific engagement with its biological target. A poor pharmacokinetic profile as a result of degradation, rapid renal clearance or nonspecific accumulation requires enabling technologies provided by delivery science. Two major delivery branches are plasma half-life extension for improved stability and prolonged plasma residence time for long-acting extracellular drugs, and targeted intracellular drug-delivery to maximize organ-specific or intracellular drug-delivery.

Poly (ethylene glycol) (PEG) conjugation, termed PEGylation [1], is the standard and predominant technology to improve solubility, stability and extend plasma half-life of peptide and protein-based drugs. PEGylation has been a highly successful approach demonstrated by an array of marketed products such as Adagen $^{\circledR}$ (PEG-adenosine deaminase [Enzon]) and Pegasys ${ }^{\circledR}$ (PEG-IFN alpha-2 $\alpha$ [Pegasys]) [2]. PEGylation, however, has drawbacks such as a need for chemical conjugation, tissue accumulation of high molecular weight PEG, and, possible reduction in efficacy and nonfavored altered drug biodistribution. From its conception from early macro and microscale systems for the controlled release of drugs, current advanced drug-delivery systems such as lipid and polymer-based nanocarriers have the capacity for temporal and spatial control of drug delivery. Exploitation of the unique properties occurring at the nanoscale has given rise to the field of 'nanomedicine' that offers great promise in the future. Nanocarrier capture by the cellular components of the mononuclear phagocyte system, immunogenicity and nonspecific tissue accumulation, however, requires surface engineering to install stealth and targeting properties. The lack of novel targeting ligands combined with elaborate design necessity and possible toxicity restricts a rapid translation into the clinic. It is clear there is a need for next-generation half-life extension and targeted drug-delivery technologies that are simple in design, biodegradable and nonimmunogenic. Human serum albumin (HSA) may provide the solution to this unmet challenge.

Albumin is a natural carrier protein possessing multiple ligand binding sites and a plasma half-life of approximately 20 days [3] facilitated by engagement with the endothelial and epithelial cellular recycling neonatal Fc receptor $\left(F_{c} R n\right)[4]$, and megalin-cubilin receptor-mediated renal rescue [5] affording a broad biodistribution and tissue penetration in normal and disease conditions. Utilization of its physiological transport mechanisms and its high charge and solubility properties, promotes albumin as a highly attractive technology that can be utilized for both half-life extension and targeted intracellular delivery applications. Its key role in ligand-binding transport for biomolecules, for example, fatty acids has been exploited in the design of albumin-binding drugs such as insulin analog detemir $\left(\right.$ Levemir $\left.^{\circledR}\right)$ [6] and

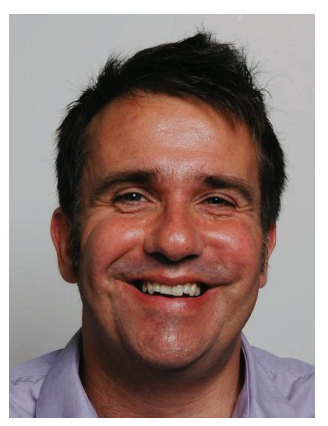

Kenneth A Howard Interdisciplinary Nanoscience Center (iNANO), Department of Molecular Biology \& Genetics, Aarhus University, 8000 Aarhus C, Denmark kenh@inano.au.dk 
glucagon-like type-1 (GLP-1) agonist liraglutide (Victoza ${ }^{\circledR}$ ) by Novo Nordisk that exhibit reversible association with endogenous albumin for long-acting effects for diabetes treatment. While, Celgene's Abraxane ${ }^{\circledR}$ is an approved nanoparticle albumin-bound paclitaxel system for the treatment of cancer [7].

\section{Inaugural albumin-based delivery technology forum}

Product advancement and a greater understanding of albumin physiology have fuelled an upsurge in albumin-based drug delivery development that was the focus of the international workshop 'Albumin: The Next Generation Protein Therapeutic' held at the 2014 Controlled Release Society Annual Meeting in Chicago, USA, 12 July 2014 [8]. The aim was to present and promote albumin-based solutions by bringing together global leaders from industry and academia to discuss current and future directions of albuminbased drug-delivery technology. Understanding albumins biological interactions is key to harness and control its drug-delivery properties; therefore, molecule binding, cellular receptor engagement, recycling and pharmacokinetics were a focus. Work from the lab of Otagiri has showed a pharmacokinetic-structural relationship of HSA genetic variants with amino acid single point mutations, and altered chain length and glycosylation that influences circulatory half-life and biodistribution $[9,10]$. This work has important implications for the tailored design of albumin-based therapies. An elegant approach toward this goal, developed between the University of Oslo and Novozymes Biopharma, is the demonstration that amino acid mutations in the FcRn binding region of engineered human albumin can be used to modulate FcRn interaction [11] and alter the blood circulation profile of albumins in rodents and nonhuman primates [12]. This is a leap forward in the field opening up the possibility to utilize a panel of recombinant albumins to control cellular interactions, pharmacokinetics and tune the therapeutic profile of drugs.

\section{"Albumin-based delivery, to some degree, has remained on the periphery of the mainstream drug-delivery community."}

The range of albumin-based drug delivery was exemplified in a session on clinical development and commercial albumin-based drug products. The availability of a single free thiol in cysteine 34 of subdomain 1A, ligand-binding sites and recombinant fusion technology allows chemical, physical and genetic engineered drug attachment strategies, respectively. Furthermore, the possibility to either utilize endogenous albumin in a 'piggy-back' approach or injectable preformulations offers remarkable pharmaceutical versatility. The long-acting insulin analog, insulin detemir, is acylated with a C14-myristic acid and liraglutide with C16-palmitic acid to physically bind in situ to circulating albumin, thus, hijack the endogenous fatty acid binding and transport process. It is thought that approximately $90 \%$ of free thiols in circulating serum proteins are contributed by HSA cysteine 34 , offering a unique target that has been exploited for site-specific chemical conjugation of cancer prodrugs. Kratz and coworkers have utilized a thiol-binding maleimide group linked to doxorubicin for in situ covalent conjugation [13]. Exploitation of the enhanced permeability and retention effect and incorporation of an acid-sensitive hydrazone spacer into the prodrug design is thought to facilitate tumor accumulation and triggered drug release in the tumor extra- and intracellular environment. This prodrug aldoxorubicin by CytRx Corporation has reached clinical trials for the treatment of Kaposi sarcoma, glioblastoma and soft tissue sarcoma. Abraxane by Celgene is a nanoparticle albumin-bound paclitaxel preformulation approved for metastatic breast cancer, non-small-cell lung cancer and metastatic pancreatic cancer. Nanoparticle disassembly into individual albumin drug-bound molecules, upon injection, results in high drug plasma levels and tumor accumulation attributed to the high metabolic uptake of albumin with a suggestion that SPARC expression in tumors is involved in an active albumin targeting process.

Genetic engineered albumin fusions offer high flexibility, ease of manufacturer and generation of single products without the requirement of drug conjugation steps or modifications. CSL Behring has an albumin fusion-based development pipeline for long-acting blood coagulation factors to treat bleeding disorders including hemophilia A and B and Von Willebrand disease. Half-life extension in humans has been demonstrated for rFIX and rFVIIa [14], toward a strategy to reduce frequency of drug infusion. Work presented showed optimization of linker spacers to reduce steric hindrance of protein activity. Albumin fusions are a growth area within the field. GlaxoSmithKline (GSK) gained approval in 2014 for albiglutide (Eper$\operatorname{zan}^{\mathrm{TM}}$ [Europe], Tanzeum ${ }^{\mathrm{TM}}$ [USA]), an albuminGLP-1 fusion as a once weekly subcutaneous injectable Type 2 diabetes mellitus treatment. EpiVax have used albumin fusion technology for IgG Fc-derived peptide Tregitopes that activate regulatory $\mathrm{T}$ cells and suppress inflammation in disease. Work presented showed Tregitope-albumin fusions inhibited ovalbumin-specific T-cell proliferation in mice. An 
EpiMatrix prediction algorithm showed no human leukocyte antigen binding or immune responses of engineered albumins that supports therapeutic utilization.

\section{Considerations \& future perspective}

The application of albumin-based solutions for halflife extension and targeted intracellular drug delivery is on the rise; demonstrated by an expanding repertoire of approved products including long-acting GLP-1 agonists Eperzan/Tanzeum (GSK), Victoza (Novo Nordisk) and insulin-analog Levemir (Novo Nordisk) for treatment of diabetes and Abraxane (Celgene) for cancer. Commercial success and increased sales growth has been achieved with 2013 sale figures for Victoza approximately $\$ 1.96$ billion (23\% sales growth) [15] and Abraxane $\$ 649$ million (52\% sales growth) [16]. Moreover, a pipeline of applications in clinical trials emphasizes a shift toward albumin research and development. Albumin is tailor-made for half-life extension applications; with its prolonged circulatory half-life, nonimmunogenic and biodegradable features. It may take time; however, to see a paradigm shift from the established PEGylation approach, however, albumin is a leading contender, with other alternative technologies early in development. A range of methods for drug attachment to albumin is available. Reports suggesting instability of maleimide in vivo [17] may require alternative coupling chemistries dependent on the stability requirements. The application of albumin-binding domain (Albumod ${ }^{\mathrm{TM}}$ [Affibody]), albumin-binding antibodies (AlbudAb ${ }^{\mathrm{TM}}[\mathrm{GSK}]$ ) and single domain nanobodies (Ablynx) attached to drugs is an exciting development. The availability of human engineered albumins with tunable engagement with recycled

\section{References}

1 Abuchowski A, McCoy JR, Palczuk NC, van Es T, Davis FF. Effect of covalent attachment of polyethylene glycol on immunogenicity and circulating life of bovine liver catalase. J. Biol. Chem. 252(11), 3582-3586 (1977).

2 Reddy K.R, Modi MW, Pedder S. Use of peginterferon alfa2a (40 KD) $\left(\right.$ Pegasys $\left.^{\circledR}\right)$ for the treatment of hepatitis C. $A d v$. Drug Deliv. Rev. 54(4), 571-586 (2002).

3 Peters, T. All about Albumin: Biochemistry, Genetics, and Medical Applications. Academic Press, MA, USA (1996).

4 Chaudhury C, Mehnaz S, Robinson JM et al. The major histocompatibility complex-related $\mathrm{Fc}$ receptor for $\mathrm{IgG}$ (FcRn) binds albumin and prolongs its lifespan. J. Exp. Med. 197(3), 315-322 (2003).

5 Koral K, Li H, Ganesh N, Birnbaum MJ, Hallows KR, Erkan E. Akt recruits Dab2 to albumin endocytosis in the proximal tubule. Am. J. Physiol. Renal Physiol. 307(12), F1380-F1389 (2014).
FcRn allows fine tuning of the therapeutic profile not currently achievable with alternative approaches. There is need, however, for development of new transgenic humanized murine models to study pharmacokinetics of these variants. Successful intracellular delivery has been demonstrated with albumin-based delivery of cancer drugs [7] but it is still unclear whether it is by passive or active targeted process and the importance of the enhanced permeability and retention effect or cellular receptors. It is paradoxical that little seems to be known about the intracellular transit pathways and nutrient release of the most abundant serum protein. Further, work on the role of FcRn and whether mechanisms have to be built into the design of the albuminbased system to escape the endosomal-dependent $\mathrm{FcRn}$ recycling process is needed to maximize an albumin-based targeted drug-delivery strategy.

Albumin-based delivery, to some degree, has remained on the periphery of the mainstream drug -delivery community. This may lie with its broad spectrum of applications and lack of dedicated forums. The Controlled Release Society workshop was a step toward this, highlighting the extensive activity and development in the field and its promotion as the vanguard for next-generation delivery technology.

\section{Financial \& competing interests disclosure}

KA Howard has no relevant affiliations or financial involvement with any organization or entity with a financial interest in or financial conflict with the subject matter or materials discussed in the manuscript. This includes employment, consultancies, honoraria, stock ownership or options, expert testimony, grants or patents received or pending, or royalties.

No writing assistance was utilized in the production of this manuscript.

6 Home, P, Kurtzhals, P. Insulin detemir: from concept to clinical experience. Expert Opin. Pharmacother. 7(3), 325-343 (2006).

7 Desai N, Trieu V, Yao Z et al. Increased antitumor activity, intratumor paclitaxel concentrations, and endothelial cell transport of cremophor-free, albumin-bound paclitaxel, ABI007, compared with cremophor-based paclitaxel. Clin. Cancer Res. 12(4), 1317-1324 (2006).

8 Controlled Release Society. www.controlledreleasesociety. org/meetings/Archives/2014AnnualMeeting/program/ Pages/Albumin.aspx

9 Iwao Y, Hiraike M, Kragh-Hansen U et al. Changes of net charge and $\alpha$-helical content affect the pharmacokinetic properties of human serum albumin. Biochim. Biophys. Acta 1774(12), 1582-1590 (2007).

10 Iwao Y, Hiraike M, Kragh-Hansen U et al. Altered chainlength and glycosylation modify the pharmacokinetics of human serum albumin. Biochim. Biophys. Acta 1794(4), 634-641 (2009). 
11 Andersen JT, Dalhus B, Cameron J et al. Structure-based mutagenesis reveals the albumin-binding site of the neonatal Fc receptor. Nat. Commun. 3, 610 (2012).

12 Andersen JT, Dalhus B, Viuff D et al. Extending serum halflife of albumin by engineering neonatal $\mathrm{Fc}$ receptor $(\mathrm{FcRn})$ binding. J. Biol. Chem. 289(19), 13492-13502 (2014).

13 Kratz F. A clinical update of using albumin as a drug vehicle - a commentary. J. Control. Release 190, 331-336 (2014).

14 Golor G, Bensen-Kennedy D, Haffner S et al. Safety and pharmacokinetics of a recombinant fusion protein linking coagulation factor VIIa with albumin in healthy volunteers. J. Thromb. Haemost. 11(11), 1977-1985 (2013).
15 Novo Nordisk. Financial Statement for 2013 (2014). www.novonordisk.com

16 Celgene Reports Fourth Quarter and Full Year 2013 Operating and Financial Results (2014).

http://ir.celgene.com

17 Tumey LN, Charati M, He T et al. Mild method for succinimide hydrolysis on ADCs: impact on ADC potency, stability, exposure, and efficacy. Bioconjug. Chem. 25(10), 1871-1880 (2014). 